\title{
Taenia solium
}

National Cancer Institute

\section{Source}

National Cancer Institute. Taenia solium. NCI Thesaurus. Code C125961.

A species of parasitic cestodes in the genus Taenia that is acquired through the ingestion of infected porcine tissue. Humans are the definitive host of the adult cestodes, resulting in taeniasis. Taenia solium also causes cysticercosis, a tissue infection caused by larval cysts, which results when humans serve as an intermediate host. 